\title{
The Brain Network in a Model of Thalamocortical Dysrhythmia
}

\author{
Mehrnoush Zobeiri, ${ }^{1}$ Gilles van Luijtelaar, ${ }^{2}$ Thomas Budde, and Ilya V. Sysoev ${ }^{3,4}$
}

\begin{abstract}
Sensory information processing and higher cognitive functions rely on the interactions between thalamus and cortex. Many types of neurological and psychiatric disorders are accompanied or driven by alterations in the brain connectivity. In this study, putative changes in functional and effective corticocortical (CC), thalamocortical (TC), and corticothalamic (CT) connectivity during wakefulness and slow-wave sleep (SWS) in a model of thalamocortical dysrhythmia, TRIP8b $\mathrm{b}^{-1-}$ mice, and in control (wild-type or WT) mice are described. Coherence and nonlinear Granger causality (GC) were calculated for twenty 10 s length epochs of SWS and active wakefulness (AW) of each animal. Coherence was reduced between 4 and ca $20 \mathrm{~Hz}$ in the cortex and between cortex and thalamus during SWS compared with AW in WT but not in TRIP8 $\mathrm{b}^{-1-}$ mice. Moreover, TRIP8b $\mathrm{b}^{-1-}$ mice showed lower CT coherence during AW compared with WT mice; these differences were no longer present during SWS. Unconditional GC analysis also showed sleep-related reductions in TC and CT couplings in WT mice, while TRIP8b ${ }^{-1-}$ mice showed diminished wake and enhanced sleep CC coupling and rather strong CT-directed coupling during wake and sleep, although smaller during sleep. Conditional GC coupling analysis confirmed the diminished CC and enhanced CT coupling in TRIP8 $\mathrm{b}^{-/-}$mice. Our findings indicate that altered properties of hyperpolarization-activated cyclic nucleotide-gated cation channels, characterizing TRIP8b ${ }^{-1-}$ mice, have clear effects on CC, TC, and CT networks. A more complete understanding of the function of the altered communication within these networks awaits detailed phenotyping of TRIP $8 \mathrm{~b}^{-l-}$ mice aimed at specifics of sensory and attentional processes.
\end{abstract}

Keywords: cortex; coupling detection; genetically modified mice; Granger causality; slow-wave sleep; thalamus

\section{Introduction}

O SCILLATORY NETWORK ACTIVITY is a characteristic property of the thalamocortical (TC) system, and is central to a number of neurocognitive and physiological functions, including cognitive processes such as attention, perception, learning and memory, arousal, and in different wake and sleep states (Steriade et al., 1993; Timofeev and Bazhenov, 2005). The different behavioral states are characterized by a specific set of TC rhythms: for instance, large amplitude $\delta$ $(0.5-4 \mathrm{~Hz})$ oscillations are the predominant oscillatory activity of the brain during deep slow-wave sleep (SWS) and spindle oscillations $(12-14 \mathrm{~Hz})$ during light SWS, while during wakefulness $\delta$ activity and sleep spindles are replaced by faster lower amplitude oscillations $(\theta, \beta$, and $\gamma)$.

The transition from the high-amplitude, low-frequency oscillations of SWS to the higher frequency, lower amplitude rhythms characterizing wakefulness creates a desynchronizing pattern on the scalp electroencephalography (EEG), and this is an emergent property of an intact TC system. This change from a synchronized to a desynchronized oscillatory pattern and vice versa is considered a basic property of all mammalian species. A number of apparently unrelated neurological and psychiatric conditions, including absence epilepsy, central tinnitus, depression, neuropathic pain, and schizophrenia, are characterized by increased low-frequency rhythmicity $(\delta$ and $\theta)$ in the TC system during states of wakefulness and were commonly termed thalamocortical dysrhythmia (TCD; Llinás et al., 1999).

TCD occurs in conjunction with a strong and common increase of TC coherence among low-frequency oscillations. This low-frequency recurrent interaction between the thalamus and cortex hampers the normal TC circuit function, and is responsible for negative and positive symptoms of the TCDs.

\footnotetext{
${ }^{1}$ Institute of Physiology I (Neurophysiology), Wesfälische Wilhelms University, Münster, Germany.

${ }^{2}$ Donders Centre for Cognition, Radboud University, Nijmegen, The Netherlands.

${ }^{3}$ Saratov State University, Saratov, Russia.

${ }^{4}$ Saratov Branch of Kotel'nikov Institute of Radio Engineering and Electronics of RAS, Saratov, Russia.

(C) Mehrnoush Zobeiri et al. 2019; Published by Mary Ann Liebert, Inc. This Open Access article is distributed under the terms of the Creative Commons Attribution Noncommercial License (http://creativecommons.org/licenses/by-nc/4.0/) which permits any noncommercial use, distribution, and reproduction in any medium, provided the original author(s) and the source are cited.
} 
The specifications of TCD in different forms of the syndrome are based on the connectivity between the involved thalamic and cortical structures (De Ridder et al., 2015; Proske et al., 2011). The abnormal slow-frequency oscillations in TCD can be caused by different mechanisms, which all result in persistent hyperpolarization of the TC relay cells (TC neurons), and a consequent increase in $\delta / \theta$ range burst activity of these neurons. The sustained hyperpolarization of TC neurons may result from inhibition of the thalamus, deafferentation, or block of ligand- or voltage-gated ion channels, including ionotropic glutamate receptor channels or hyperpolarization-activated cyclic nucleotide-gated cation (HCN) channels (Chung et al., 2009; Llinás et al., 2005, 1999; Ludwig et al., 2003; Zhang et al., 2009). The latter is known to contribute in the generation of the slow TC oscillations. In fact, the cyclic interaction between $\mathrm{HCN}$ channels and T-type $\mathrm{Ca}^{2+}$ channels in TC neurons constitutes the basis for slow oscillations (Fogerson and Huguenard, 2016).

Recently, we introduced the tetratricopeptide repeatcontaining Rab8b-interacting (TRIP8b) protein, a brain-specific auxiliary subunit of HCN channels, as a molecule contributing to the modulation of physiological TC oscillations. The presence of this protein has direct effect on HCN channel expression in both thalamus and cortex (Heuermann et al., 2016; Zobeiri et al., 2018). We showed that in TRIP8b-deficient mice (TRIP8 $\mathrm{b}^{-1-}$ ) downregulation of hyperpolarizationactivated current $\left(\mathrm{I}_{h}\right)$ in cortex and thalamus, as well as decreased cyclic adenosine monophosphate (cAMP) signaling, is responsible for transformation of the normal physiological sleep- and wake oscillations to a new form of TCD characterized by a significant increase in $\delta$ oscillations during episodes of AW and a lack of the natural desynchronizing response of the EEG in transition from deep SWS to AW.

Interestingly, these findings pointed to the reduction of $\mathrm{HCN}$ channel function as an alteration associated with TCD (Chung et al., 2009; Datunashvili et al., 2018; Heuermann et al., 2016; Ludwig et al., 2003; Zobeiri et al., 2018) and opened up the possibility to assess possible additional changes in network function. While a number of molecular alterations leading to TCD have been identified, changes in network connectivity supporting TCD have been addressed to a lesser extent and largely depend on computational modeling analysis (Proske et al., 2011). Since understanding the nature of the networks involved in TCD is an important step in developing new therapeutic approaches for a wide range of TCD-related neurological and psychiatric disorders, we compared the brain functional connectivity in $\mathrm{TRIP}^{-1-}$ mice with the commonly used wild-type (WT; C57BL/6J) controls. More specifically, we investigated the functional corticocortical (CC), corticothalamic (CT), and TC connectivity during AW and during SWS.

Two different approaches were used here to describe network activity from local field potentials (LFPs). First, coherence functions were calculated, providing a first linear frequency-specific estimation of whether the networks differ between normal and genetically modified mice. The simplest generalization of the coherency function for a multivariate case, allowing detection of directed coupling, is partial directed coherence (Baccalá and Sameshima, 2001) or its renormalized version (Schelter et al., 2009). However, considering that coupling between different regions might not be linear, nonlinear Granger causality (nIGC) method (Chen et al., 2004) with specific adaptations to LFP (Kornilov et al., 2016; Sysoeva and Sysoev, 2012) was applied, followed by a multivariate variant of GC.

The nlGC approach has been previously used to describe the dynamics of coupling periictally in rats with a genetic form of absence epilepsy (Sysoeva et al., 2016b). Coherence and nlGC were calculated between the motor cortex $(\mathrm{MoCx})$ and somatosensory cortex (SoSeCx), and between the $\mathrm{SoSeCx}$ and lateral dorsal thalamus.

\section{Materials and Methods}

\section{Animals}

All experimental procedures on TRIP8 $\mathrm{b}^{-/-}$and control mice were performed in accordance with the principles approved by local authorities (review board institution: Landesamt für Natur, Umwelt und Verbraucherschutz Nordrhein-Westfalen; approval IDs: 84-02.04.2015.A574, 84-02.05.50.15.026) and described earlier (Zobeiri et al., 2018). Efforts were made to minimize the number of animals and the degree of discomfort to animals used in this study.

\section{Electrode implantation and LFP recordings}

Three- to five-month-old male TRIP8b ${ }^{-1-}$ and WT mice were used; the same animals as previously described, also procedures for electrode implantation, LPF recording, and selection of the LFP epochs were described before (Zobeiri et al., 2018). In brief, animals were anesthetized; analgesia was applied before surgery for stereotactical implantation of cortical and thalamic electrodes. Isolated (except at the tip) stainless steel electrodes were implanted: SoSeCx: A/ $\mathrm{P}=0, \mathrm{M} / \mathrm{L}=3$, depth $=-1.2 ;$ MoCx: $\mathrm{A} / \mathrm{P}=2, \mathrm{M} / \mathrm{L}=1.5$, depth $=-0.8$; and ventral posterior medial nucleus of the thalamus (VPM) nucleus: $\mathrm{A} / \mathrm{P}=-1.7, \mathrm{M} / \mathrm{L}=1.5$, depth $=-2.8$. Coordinates were according to the mouse stereotaxic coordinates of Paxinos and Franklin. Two epidural silver wires on top of the cerebellum served as ground and reference electrodes, respectively. The electrode assembly was fixed to the skull with dental acrylic cement. Mice were allowed to recover for at least 1 week.

Differential LFP signals from the two cortical sites and thalamus were recorded continuously for $8 \mathrm{~h}$ starting at 8 am during the light phase of the 12-12 h light-dark cycle in freely moving mice. The LFP signals were amplified, filtered by a band-pass filter, and only frequencies between 1 and $100 \mathrm{~Hz}$ were allowed to pass. Digitizing occurred with a sampling rate of $f_{m}=1042 \mathrm{~Hz}$ by a Cambridge Electronic Design, United Kingdom recording system. The behavioral activity of the animals was registered by a Passive Infrared Registration System (PIR, RK2000DPC LuNAR PR Ceiling Mount, Rokonet RISCO Group S.A., Drogenbos, BE).

LFP data from the two cortical sites and thalamus from 20 epochs of $10 \mathrm{~s}$ of AW and deep SWS were selected for each animal based on the LFP and PIR activity according to commonly used criteria (van Luijtelaar et al., 2012; Zobeiri et al., 2018). Only epochs from the first $2 \mathrm{~h}$ of the light period were selected; these hours represent the periods with the largest amount of specifically deep SWS (Huber et al., 2000). SWS criteria are provided as follows: high-amplitude cortical LFP together with slow $(1-4 \mathrm{~Hz})$ waves in a motionless animal (low-amplitude, stable, regular PIR signal); AW 
criteria: behavioral activity (high and variable PIR signal accompanied by low-amplitude cortical LFP with $\theta$ and/or $\beta$ ) for the WT mice; in TRIP8b ${ }^{-1-}$, the PIR signal was only used since a dissociation between LFP and behavior was noticed (Zobeiri et al., 2018), and a high amplitude and changing PIR signal was the only criterion used to establish AW.

After the LFP recordings, animals were deeply anesthetized with isoflurane; a direct current of $9 \mathrm{~V}, 25 \mu \mathrm{A}, 10 \mathrm{~s}$ duration was passed through each electrode. Brains were removed, and post fixed overnight in $4 \%$ paraformaldehyde and later in $30 \%$ sucrose for $48-72 \mathrm{~h}$. Free-floating coronal sections $(40 \mu \mathrm{m})$ were cut with a microtome and stained with cresyl violet. Only animals with confirmed electrode positions were included in the data selection and analysis; five TRIP8 $\mathrm{b}^{-1-}$ and control mice were included in the analyses.

\section{Coherence function}

Coherence function $C_{x y}$ is a classical tool to study linear, frequency-resolved similarities of two signals $\left\{x_{n}\right\}_{n=1}^{N}$ and $\left\{y_{n}\right\}_{n=1}^{N}$. In contrast to the cross-spectrum, coherence function is normalized in the range $0 \leq C_{x y} \leq 1$ and is independent of the amplitude of the signals.

Coherence functions were calculated using Equation (1), given $K$ segments of both time series (LFP signals) $\left\{x_{n}\right\}_{n=1}^{N}$ and $\left\{y_{n}\right\}_{n=1}^{N}$, each segment consisting of the same number of samples $N$, and sampling rate $f_{m}$. Usually, the higher $K$ is, the lower coherence values can be detected. Values under the $1 / \sqrt{(K)}$ threshold are mostly considered meaningless, since they cannot be distinguished from 0 :

$$
\begin{aligned}
C_{x y} & =\frac{\left|S_{x y}(f)\right|}{\sqrt{S_{x x}(f) S_{x y}(f)}}, \\
S_{x x}(f) & =\left|F_{x}(f)\right|_{k=1}^{2 K}, \\
S_{y y}(f) & =\left|F_{y}(f)\right|_{k=1}^{2 K}, \\
S_{x y}(f) & =F_{x}(f) \cdot F_{y}(f)_{k=1}^{K} .
\end{aligned}
$$

Equation (1) was used to calculate coherence for each mouse for both states (AW and SWS) for both channel pairs $(\mathrm{MoCx}-\mathrm{SoSeCx}$ and $\mathrm{SoSeCx}-\mathrm{VPM})$ separately using $K=20$ episodes of the same length of 10 s $(N=10416$ data points). The Fast Fourier transform from the scientific package NumPy (van Der Walt et al., 2011) was used.

Differences in coherence values for the two strains of mice and between two states of vigilance were evaluated by Student's $t$-tests (two sided) for each frequency $(1-100 \mathrm{~Hz}$ ) using scipy.stats scientific package. To correct for multiple comparisons (i.e., different frequencies), nearby values were considered as triplets following Maris and Oostenveld (2007), and Bonferroni correction was applied. This approach reduced the frequency resolution to $0.3 \mathrm{~Hz}$.

\section{Granger causality}

NlGC with polynomial nonlinearity (Chen et al., 2004) was used as a primary tool for directed coupling analysis. This approach is based on the construction of a forecasting model. The normalized prediction improvement $(0 \leq P I \leq 1)$ is the main dependent variable: it characterizes how much information from one signal is incorporated into the prediction of a second signal. The Bayesian information criterion (BIC) was used to estimate the dimension and polynomial order of the most optimal prediction model (Schwarz, 1978). Nonuni- form embedding was used for vector-state reconstruction to reduce the number of coefficients to be estimated in the model (Kugiumtzis, 1996). Following the results of BIC analysis and recommendations for accounting time scales (Kornilov et al., 2016), different models were used for sleep and wakefulness. Therefore, the PI values comparing sleep and wakefulness should be interpreted with some caution.

Functional connections between MoCx and $\mathrm{SoSeCx}$ and between $\mathrm{SoSeCx}$ and VPM were pairwise studied, independently in both directions (so four couplings were analyzed). It needs to be remarked that mediated coupling between MoCx and VPM cannot be completely excluded with this pairwise approach, also considering that there are weak anatomical connections between MoCx and VPM. But this indirect coupling cannot effect the results significantly, since first, these anatomical couplings are weak, and second, it was shown that the applied method is not very sensitive to mediated coupling in the presence of a direct one (Sysoev and Sysoeva, 2015).

The currently used nlGC method can account for the nonlinearity in the signals as well as their nonlinear coupling. However, the nlGC method is mainly applicable if the length of the time series is larger than the length necessary for the linear variant (Chen et al., 2004); otherwise, the number of coefficients in the prediction models would be too high to reliably estimate the model coefficients.

The above-described bivariate unconditional nlGC method assumes that all detected couplings are direct. Although the brain areas analyzed here are for sure directly connected through reciprocal structural connections, it is not certain that the communication might not involve a third node. Therefore, a conditional (multivariate) GC was additionally used (see Appendix) to determine which couplings detected by unconditional nlGC are direct and which are mediated. In the results and discussion, we focus on the common findings as obtained with the unconditional and conditional nlGC method, without ignoring the discrepancies.

There are two possibilities to evaluate statistically the putative differences between and within the TRIP8b ${ }^{-1-}$ and WT mice obtained using nlGC analyses: analytical tests (Smirnov et al., 2013) and testing against surrogate data (Theiler et al., 1992). The latter is possible since we have episodes of exactly the same length for each animal, and surrogate data can be constructed by switching channels: taking one channel from one episode and the other channel from another episode. This approach has an advantage over most analytical approaches; for example, the one pointed by Smirnov et al. (2013), since it demands no additional assumptions about the data being analyzed or about the distribution of model's residuals. Tests against surrogate data show a higher specificity in comparison with some other popular approaches (Kornilov and Sysoev, 2013), producing less false-positive detections of bidirectional coupling from time series of actually unidirectionally coupled systems.

All possible surrogates ( 380 per animal for each channel combination for sleep and wake separately) were constructed, and nlGC was calculated for all of them. Theoretically, using the surrogates where the driving subsystem episode precedes the driven subsystem episode is dangerous, since some causality can originate from this. However, the Largest Lyapunov exponent for typical LFP data was shown to be very high (e.g., see Medvedeva et al., 2016), 
making forecast with times $>2 \mathrm{~s}$ impossible by the commonly used type of models. Our episodes were separated by time intervals of at least $10 \mathrm{~s}$. In addition, the Kolmogorov-Smirnov test for distribution of surrogate PI calculated using preceding episodes of the driving subsystem and its postponed episodes showed that these distributions are completely indistinguishable in all cases.

The highest surrogate $P I$ was considered as a threshold of significance with $p$-value $p=1 / 381 \approx 0.00262$. Then, the number of significant outcomes $A$ was calculated for all five mice together (per group) per channel pair and per direction in each experiment separately based on the surrogate data. $A$ is in the range of $0-100$ ( 5 animals $\times 20$ episodes per animal $)$; therefore, $A$ also can be considered as a percentage. Also, the number $A$ can be considered as an indicator of coupling strength, if it is not always very low (near 0) or very high (near 100). The surrogate analysis was done independently per animal for each channel pair in each state and direction. Since surrogate data completely reproduce all features of real pairs except coupling (they are uncoupled, being taken from episodes distinct in time), they include all signal peculiarities.

The $p$-value $p \approx 0.00262$ is the significance level for a single outcome. Here, we deal with multiple (100) tests for each of the considered cases; therefore, it is imperative to correct for these multiple tests. Since our goal is to compare coupling for different cases and different channel pairs, the value of $\Delta A_{\text {critical }}=\left|A_{1}-A_{2}\right|$ was considered as the minimal absolute difference for a significant difference between strains, states, or direction of coupling of different channel pairs:

$$
\begin{aligned}
& p^{\prime}\left(A_{1}, A_{2}\right)=2 \cdot 2 \cdot 4 \cdot 5 \cdot 20 \\
& \cdot \sum_{i=a}^{b}\left(C_{b}^{a} \cdot p^{b-i} \cdot(1-p)^{i} \cdot C_{100-a}^{i-a} \cdot p^{i-a} \cdot(1-p)^{100-a+i}\right), \\
& b=\max \left(A_{1}, A_{2}\right), a=\min \left(A_{1}, A_{2}\right) \text {, } \\
& C_{b}^{a}=\frac{b !}{a ! \cdot(b-a) !}
\end{aligned}
$$

where 20 stands for the number of episodes, 5 for the number of animals, 4 for the number of analyzed channel pairs (considering different directions for the same channels as a separate pair), 2 for the two types of animals, and the other 2 for the two states (sleep and wake), $A_{1}$ and $A_{2}$ are the numbers of significant coupling outcomes, $a$ ! is the factorial of $a, C_{b}^{a}$ is the number of combinations from $b$ by $a$.

Equation (2) originates from the binomial distribution for $p$, with the simplification for the case of $p$ not close to 1 (in our case, this was always true). One can easily find that Equation (2) provides the cumulative corrected $p$-value $p^{\prime}<0.0144$ for any $A_{1}$ and $A_{2}$ if $\left|A_{2}-A_{1}\right| \geq 5$; that is, $\Delta A_{\text {critical }}=5$ is significant on the generally accepted level, while difference in four can have $p^{\prime} \approx 0.25$, which is not usually accepted. $\Delta A_{\text {critical }}=6$ has $p^{\prime}<6.7 \cdot 10^{-4}$ and $\Delta A_{\text {critical }}=7-p^{\prime}<2.7 \cdot 10^{-5}$.

\section{Results}

\section{Coherence analyses}

Coherence analyses were performed on the LFP data recorded from three channels (we used two channel pairs:
MoCx-SoSeCx and SoSeCx-VPM) for two stages of vigilance and two strains, considering they have well-described reciprocal anatomical connections. The figures show the mean of five animals per strain; comparing AW and SWS within and (Figs. 1 and $2 \mathrm{a}, \mathrm{b}$, respectively) between the two strains (Figs. 1 and 2c, d).

$\mathrm{CC}$ functional connectivity during AW and SWS. Figure 1 illustrates the coherence function between the two cortical channel pairs: MoCx and SoSeCx, in WT and TRIP8b ${ }^{-1-}$ mice during AW and SWS. Clear differences between sleep and wake in coherence were noticed in the WT mice, with a significant reduction between 4 and $19 \mathrm{~Hz}$ (Fig. 1a), in agreement with previous results observed in rats $(\mathrm{Pal}$ et al., 2016). Interestingly, this reduction was not present in TRIP8b ${ }^{-1-}$ mice (Fig. 1b). Instead, a nonsignificant reduction could be noticed from 4 to $\sim 11 \mathrm{~Hz}$ and $\sim 60 \mathrm{~Hz}$. In other words, in TRIP8b ${ }^{-1-}$ mice the functional CC network connectivity during wakefulness resembles the network connectivity during sleep. From the data presented in Figure 1c, $\mathrm{d}$, one can see that there is no difference in coherence between WT and TRIP8b ${ }^{-1-}$ mice during both AW and SWS.

CT functional connectivity during AW and SWS. The coherence analyses between the SoSeCx and VPM are presented in Figure 2. As illustrated in Figure 2a, WT mice showed significantly lower cortical thalamic coherence levels in frequencies between 5-17 and also $19 \mathrm{~Hz}$ during SWS compared with AW. The coherence was in general lower in TRIP8b $b^{-1-}$ mice compared with WT. The differences observed in coherence between SWS and AW in $\mathrm{TRIP} \mathrm{b}^{-1-}$ mice were marginal and seemed to be shifted to the lower frequencies (Fig. 1b). No significant differences in coherence were observed during SWS between the two strains (Fig. 2d). In contrast, TRIP8b ${ }^{-1-}$ mice showed significantly lower coherence levels between cortex and thalamus during AW when compared with WT mice (Fig. 2c).

\section{Coupling analyses using n/GC}

Coherence shows only linear similarities of signals, and it cannot resolve the coupling direction. To address both of these issues, we compared first unconditional directed coupling using nlGC, as explained earlier, between strains and states. Later, this was followed by the conditional variant. Couplings higher than $\Delta A_{\text {critical }}=5$ were considered as being significant, also differences between groups and states $\geq 5$. The same channel pairs were investigated as with coherence analyses.

CC effective connectivity during AW and SWS. First, the cortical couplings in WT mice were established using pairwise (unconditional) approach; for results, see Figure 3a. The interconnections during wakefulness between $\mathrm{MoCx}$ and SoSeCx were asymmetric: a dominating drive from MoCx to SoSeCx (14), the reversed drive was 9. The coupling during sleep from $\mathrm{SoSeCx}$ to $\mathrm{MoCx}$ remained the same (9-9) as during wakefulness; the coupling from $\mathrm{MoCx}$ to $\mathrm{SoSeCx}$ reduced insignificantly (from 14 to 10 ). This implied that the intracortical interactions became more symmetrical (10 and 9) during sleep. The results of the conditional approach (presented in Fig. 4) did not differ 


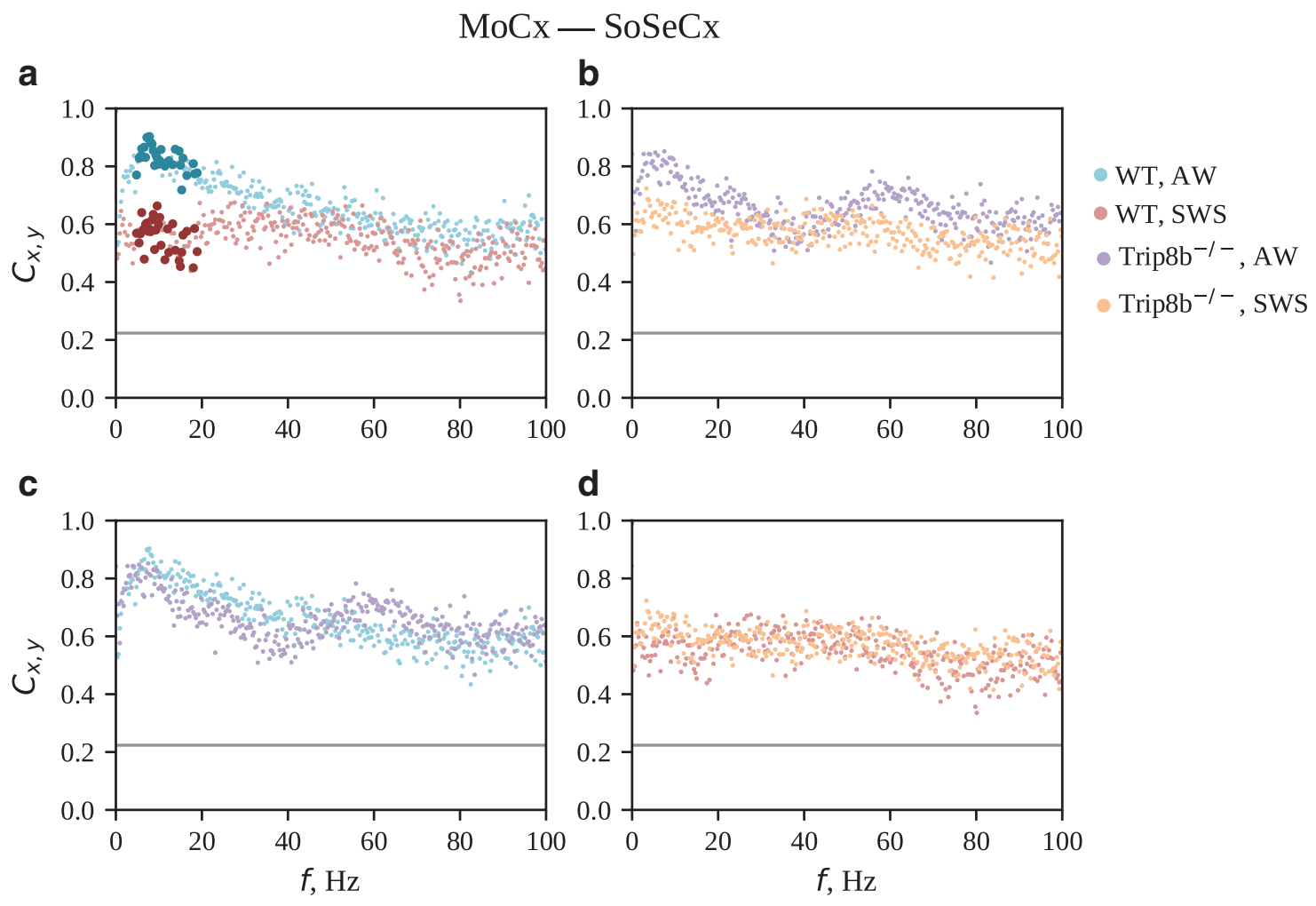

FIG. 1. The coherence function for channel pair MoCx-SoSeCx averaged over five animals. Plots (a) and (b) compare the cortical-thalamic coherence during SWS and AW in WT and TRIP8b ${ }^{-1}$ mice, respectively. Note (in a) the significantly (bold circles) lower coherence levels in WT mice in frequencies between 4 and $19 \mathrm{~Hz}$ during SWS compared with AW and a lack of statistical differences in TRIP8b $b^{-/-}$mice (b). In plots (c) and (d), the coherence is compared between the two strains during AW and SWS, respectively. The gray horizontal lines indicate the level, under which the coherence is considered unreliable due to insufficient averaging. AW, active wakefulness; MoCx, motor cortex; SoSeCx, somatosensory cortex; SWS, slow-wave sleep; WT, wild type.

much from the pairwise approach results, except that the reduction in coupling during SWS in comparison with AW in the direction MoCx $\rightarrow$ SoSeCx, which was also obtained by the unconditional nlGC method, became significant.

$\mathrm{TRIP}^{-1-}$ mice also showed asymmetric cortical coupling during wakefulness (6 vs. 1) based on pairwise analysis, although the strength of couplings was less than that in WT mice (14 and 9), and in particular the drive from MoCx to SoSeCx was missing. Both cortical couplings increased during sleep in both directions from 1 to 10 for SoSeCx to MoCx and from 6 to 15 in the opposite direction. This increased driving from MoCx to SoSeCx during sleep in TRIP8b ${ }^{-1-}$ mice was not confirmed with the conditional analysis. These CC couplings in TRIP8b ${ }^{-1-}$ mice were low during wakefulness and remained low during SWS, especially the couplings between SoSeCx and MoCx during wakefulness and sleep were lower in TRIP8b ${ }^{-1-}$ than in the WT mice.

Corticothalamo-cortical effective connectivity during AW and SWS. CT and TC conditional and unconditional coupling estimates during wakefulness in WT mice were present and significant in both directions. Sleep reduced the strength of the coupling in both directions, and this was significant for the unconditional analysis. In contrast, TRIP8b ${ }^{-1-}$ mice showed a significant and much stronger CT coupling based on both unconditional and conditional analyses during both AW and SWS compared with WT mice, while the reversed couplings were not so extreme based both on conditional and unconditional approaches, although that TRIP8b ${ }^{-1-}$ mice showed a lower TC bivariate coupling strength during wakefulness compared with WT mice (10 vs. 19). All couplings between cortex and thalamus were reduced during sleep in TRIP8 $b^{-1-}$ mice as shown by both conditional and unconditional methods. The results of the unconditional analyses are depicted in Figure 3; the ones from the conditional analysis shown in Figure 4.

\section{Discussion}

This study sought to understand the possible alterations in functional brain connectivity in TRIP $8 \mathrm{~b}^{-1-}$ mice, which have previously been introduced as a model of TCD (Zobeiri et al., 2018). CC, TC, and CT network analyses with two different methods, namely coherence and nlGC, showed unambiguous changes in functional and effective brain connectives in TRIP8 $\mathrm{b}^{-T-}$ compared with WT mice. The major findings of this study are as follows:

1. Application of coherence function in a CC and cortical and thalamic channel pair in WT mice showed a clear sleep-induced reduction in coherence in frequencies between 4 and $19 \mathrm{~Hz}$ (high $\delta$ to low $\beta$ frequency 


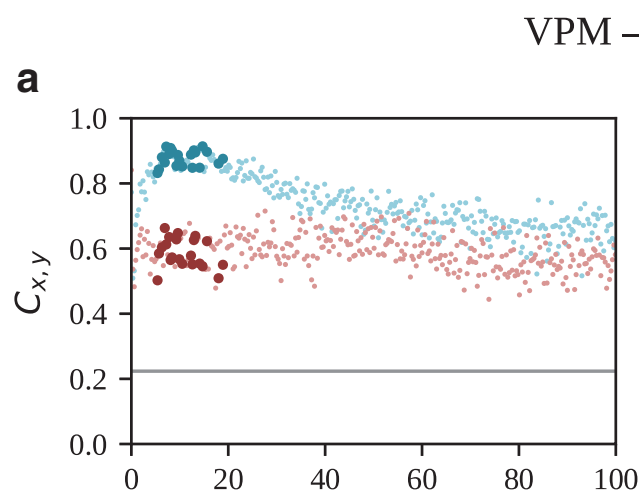

- SoSeCx

b

C
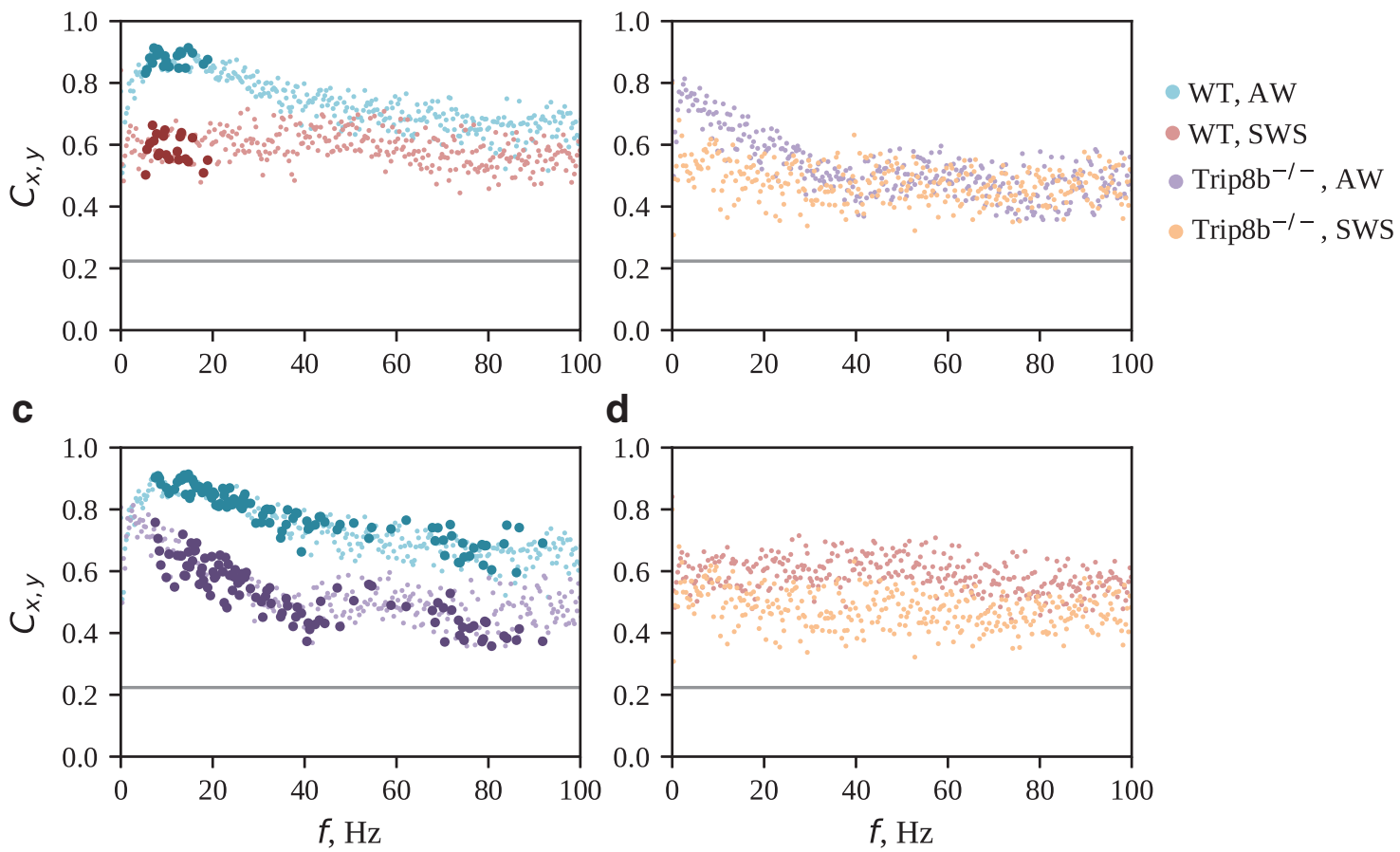

FIG. 2. The coherence function for channel pair SoSeCx-VPM averaged over five animals. Plots (a) and (b) compare the cortical-thalamic coherence during SWS and AW in WT and TRIP8b ${ }^{-1-}$ mice, respectively. As shown in (a), WT mice show a significantly (bold circles) lower cortical thalamic coherence levels during SWS compared with AW. In plots (c) and (d), the coherence is compared between the two strains during AW and SWS, respectively. Note (in c) the significantly lower cortical thalamic coherence levels during AW in TRIP8b ${ }^{-1-}$ compared with WT mice. The gray horizontal lines indicate the level under which the coherence is considered as unreliable due to insufficient averaging. VPM, ventral posterior medial nucleus of the thalamus.

bands). The sleep-induced decrease in coherence was not present in TRIP8b ${ }^{-1-}$ mice.

2. TRIP8b ${ }^{-1-}$ mice showed a substantially lower cortical thalamic coherence in a wide frequency range during wakefulness when compared with WT mice.

3. Coupling analysis in the WT mice showed a sleepinduced reduction in coupling mainly between CT and $\mathrm{TC}$ regions.

4. TRIP8b ${ }^{-l-}$ mice showed a rather strong CT coupling during both wakefulness and sleep compared with WT mice, with a lower coupling strength during sleep. The coupling in the opposite direction in TRIP8 $\mathrm{b}^{-\gamma-}$ mice, that is, from thalamus to cortex, was not exceptionally high and also lower during sleep.

5. TRIP8b ${ }^{-1-}$ mice showed less CC coupling during wakefulness compared with WT mice.

\section{CC connectivity during wakefulness and SWS}

EEG coherence provides valuable information about the functional interactions between two or more neural networks, and is used for detection of the network synchrony based on frequencies of neuronal oscillations. As compared with AW, SWS in WT mice was characterized by a decrease in $\mathrm{CC}$ and also cortical thalamic coherence in frequencies between 4 and $19 \mathrm{~Hz}$ (high $\delta$ to medium $\beta$ ). These results were in line with the outcome of a previous study on rats in which a significant reduction in the CC coherence levels in $\delta, \theta$, and $\alpha(0.5-15 \mathrm{~Hz})$ bands was found (Pal et al., 2016; White et al., 2011). However, analyses of sleep EEG in healthy human individuals indicated an increase in intrahemispheric coherence in the spindle $(7-12 \mathrm{~Hz})$ band and decrease in other frequencies mainly from 20 to $45 \mathrm{~Hz}$, when comparing rapid eye movement (REM) sleep with non-REM sleep and without including baseline wakefulness reference values (Achermann and Borbély, 1998).

Intrahemispheric CC correlation studies on human subjects comparing SWS and wakefulness EEG signals revealed similar results to those found in the Achermann and Borbély study; a higher correlation in the range of sleep spindles and $\alpha 2$ frequencies during SWS compared with wakefulness (Guevara et al., 1995). The discrepancies between human and rodents coherence studies might stem from the differences in the brain structural and functional connectivity of the two species, the anatomical organization of the brain, differences between EEG and LFP recording procedures per se, or simply from differences in wake state reported in human (the resting-wake EEG) studies and AW in our study.

Striking was the lack of differences between the corticalthalamic and CC coherence during wake and sleep episodes in TRIP8b ${ }^{-1-}$ mice. Although coherence is obviously not an estimate of EEG power, comparing the power spectral density of the cortical and thalamic LFP in wake and 
a AW
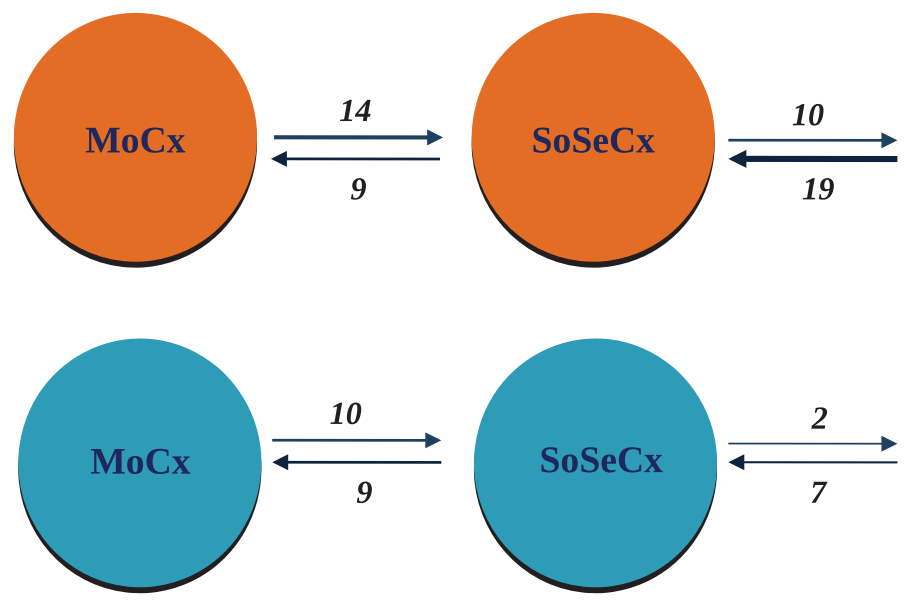

b

AW

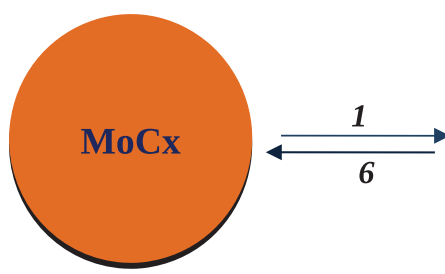

TRIP8b $^{-/-}$
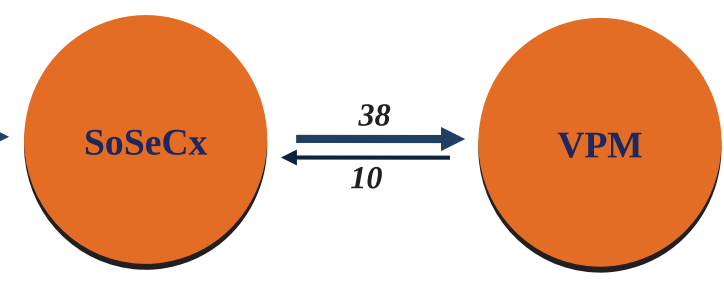

29
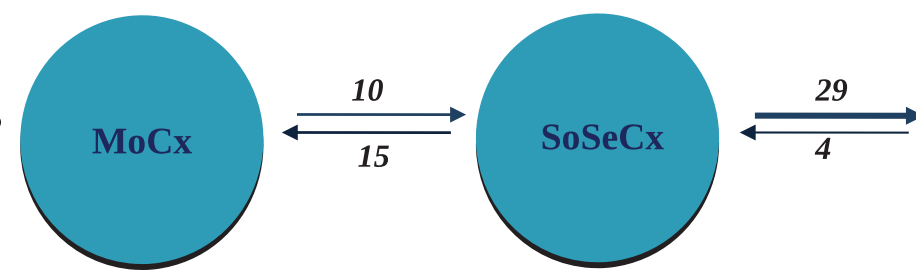
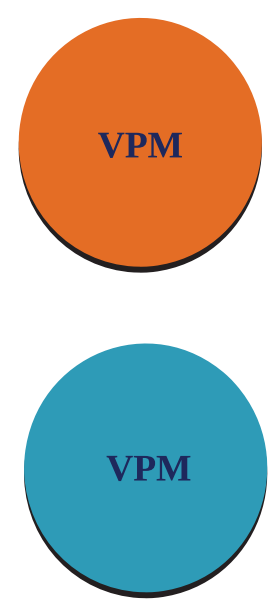

FIG. 3. Results of unconditional coupling analysis using nlGC. Arrow heads define the direction of the coupling, and numbers show the numbers (and percentage) of significant couplings $A$ in WT (a) and TRIP8b ${ }^{-1-}$ (b) mice during AW and SWS. Difference $\geq 5$ is considered as significant at the level 0.05 . The thickness of the arrows corresponds to the numbers; the thicker the arrow, the larger the number of significant couplings found in the corresponding direction. nlGC, nonlinear Granger causality. sleeping TRIP8b ${ }^{-1-}$ mice in our previous study (Zobeiri et al., 2018) also revealed smaller differences between the two stages of vigilance.

In addition, analysis of CC-directed coupling using both unconditional and conditional approaches in TRIP8 $\mathrm{b}^{-1-}$ mice revealed a low level of connectivity between $\mathrm{SoSeCx}$ and $\mathrm{MoCx}$ during $\mathrm{AW}$ in comparison with the control mice. Although the exact mechanism for the lower CC coupling in TRIP8b ${ }^{-1-}$ mice is not clear, the bidirectional (hypo- or hyperconnectivity) region-specific alterations in functional connectivity have been reported in other TCDs (Zhang et al., 2012), and might result from both differences in anatomical connections between cortical regions and variations in the sources of thalamic inputs (inputs from higher- vs. first-order thalamic nuclei) that each specific cortical region receives. The latter is of great importance, considering that many CC communications go through the higher order thalamic nuclei (Sherman, 2017). Interestingly, in addition to the ventral-basal complex of the thalamus, TRIP8b ${ }^{-1-}$ mice show similar alterations in the HCN channels expression and activation kinetics in some other thalamic nuclei, including posterior-thalamic nucleus (PO; Zobeiri et al., 2018), which is considered as a higher order nucleus innervating the MoCx. All adds to a suspected complex pattern of functional alterations in the brain networks in TRIP8b $\mathrm{b}^{-1-}$ mice, which may influence sensory processing, sensorymotor functions, and arousal reactions.

\section{TC connectivity during AW and SWS}

The interaction between thalamus and neocortex underlies many important brain processes such as relay of sensory signals during wakefulness, diminished sensory transmission, and increased sensory awareness thresholds during sleep (Llinas and Steriade, 2006; Picchioni et al., 2014; Steriade et al., 1993). The traditional view explaining reduced brain responsiveness during SWS is that a thalamic gate disrupts signal transmission from the periphery to cortex (Coenen and Vendrik, 1972; Steriade et al., 1990). This reduction of information transfer has been established to be present first for the visual modality, subsequently also for the other sensory modalities. The reduction in the functional TC connectivity from wakefulness to sleep has been reported in several studies (Picchioni et al., 2014; Spoormaker et al., 2010). The lower functional connectivity between the thalamus and cortex during sleep is to a higher extent due to the changes in firing pattern and intrinsic properties of TC neurons (Steriade et al., 1993). Analysis of the coherence between SoSeCx and 
a

AW
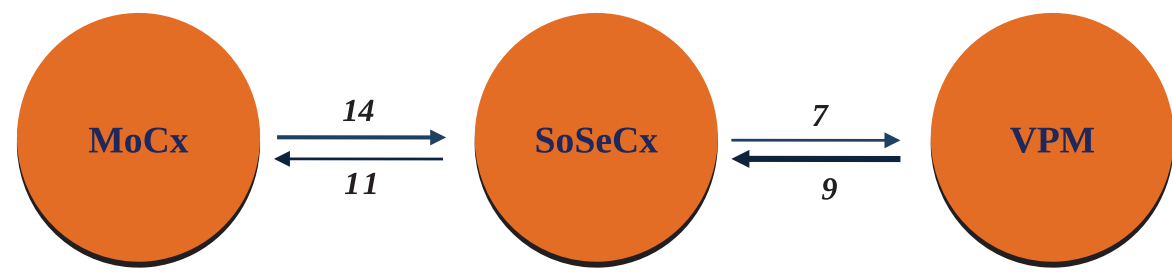

FIG. 4. Results of coupling analysis using conditional nlGC. Arrow heads define the direction of the coupling, and numbers show the numbers (and percentage) of significant couplings $A$ in WT (a) and TRIP8b ${ }^{-1-}$ (b) mice during AW and SWS. Difference $\geq 5$ is considered as significant at the level 0.05 . The thickness of the arrows corresponds to the numbers; the thicker the arrow, the larger the number of significant couplings found in the corresponding direction.
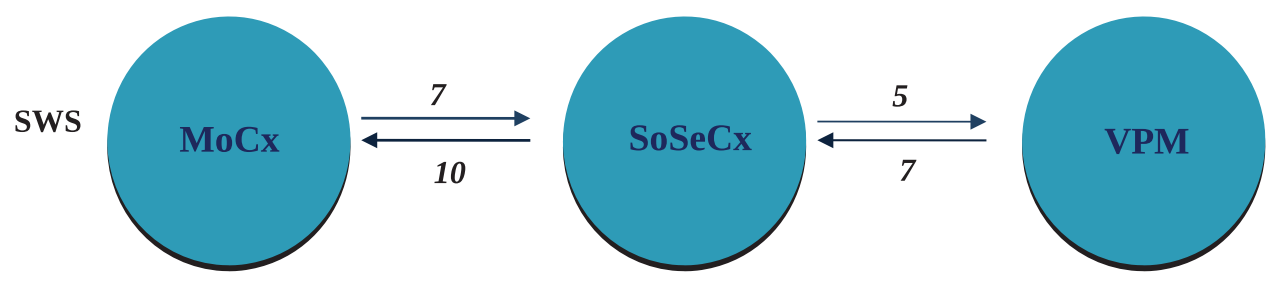

b

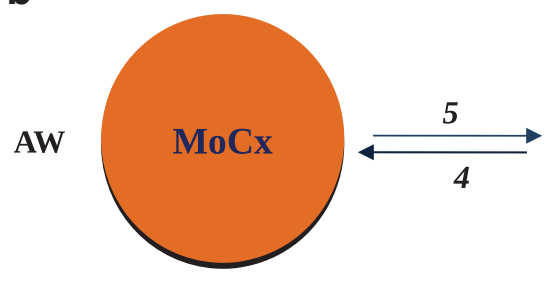

TRIP8b $^{-/-}$

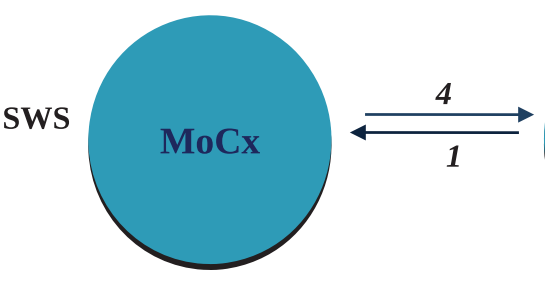

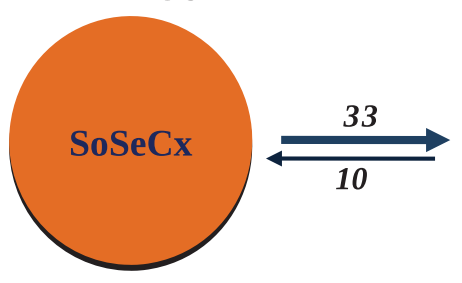
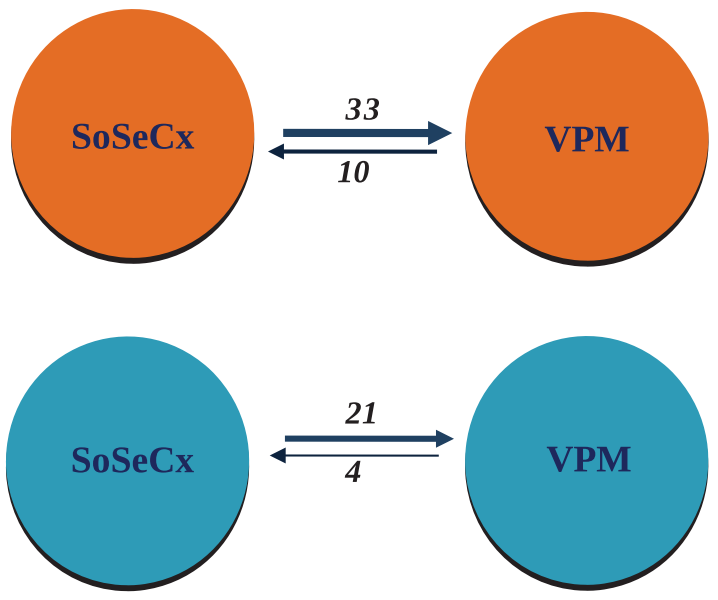

VPM in our study revealed a significant reduction in the coherence levels of a wide range of frequencies in TRIP8 $\mathrm{b}^{-/-}$ mice compared with WT animals during AW, indicating lack of synchronous activity and communication between these two regions.

Moreover, coherence and directed conditional and nonconditional coupling analyses in TRIP8 $\mathrm{b}^{-1-}$ mice showed an unbalanced pattern of connectivity between cortex and thalamus; strong directed coupling from SoSeCx to VPM and a much lower level of coupling from VPM to SoSeCx. A further reduction in functional coupling was found in TRIP8 $\mathrm{b}^{-1-}$ mice between thalamus and cortex during sleep, reflecting the lower sensory information flow from VPM to SoSeCx.

Abnormalities in motor/somatosensory-thalamic connectivity have been reported in some neurobiological disorders such as schizophrenia and autism (Woodward and Cascio, 2015). Using resting-state functional magnetic resonance imaging, Woodward et al. (2012) showed differential abnormalities of TC networks in schizophrenia with reduced prefrontal-thalamic connectivity and increased motor/ somatosensory-thalamic connectivity (Woodward et al., 2012). Considering that schizophrenia has been classified as a TCD, our results may suggest the existence of similar changes in brain connectivity between different types of TCDs. Whether the changes observed in the functional connectivity between the thalamus and cortex might affect specific sensory-related cognitive processes should be addressed in the future.

\section{Relationship between abnormal brain connectivity in TRIP8b ${ }^{-/-}$mice and HCN channels dysfunction}

The role of hyperpolarization-activated current $\left(I_{h}\right)$ in single cell and TC network oscillations during sleep and wakefulness, and in memory and cognitive processes has been extensively studied (He et al., 2014). Dysregulation of $I_{h}$ has been linked to a number of neurological disorders with concomitant changes in TCT oscillatory activities such as in certain types of epilepsy (Cain et al., 2015; Heuermann et al., 2016; Kole et al., 2007). In our previous study, we showed that downregulation of $\mathrm{I}_{\mathrm{h}}$ in cortical pyramidal and TC neurons of TRIP8 $\mathrm{b}^{-1-}$ mice is a cause factor for changes in physiological TC oscillations. It is known that the firing pattern of TC neurons at the transition from wake to light SWS changes from tonic to phasic due to the hyperpolarization of these neurons induced by inhibitory GABAergic inputs from the thalamic reticular nucleus (TRN), resulting in generation of slow waves and sleep spindles. As sleep deepens and TC neurons become further hyperpolarized, $\delta$ oscillations appear on cortical EEG and thalamus, and the 
neocortex becomes functionally disconnected (Steriade et al., 1993; Timofeev et al., 1996).

The lower functional connectivity of thalamus and cortex during SWS is accompanied by an increase in sensory awareness threshold. In our previous study, we showed that reduction of $I_{h}$ in TC neurons of TRIP8b ${ }^{-1-}$ mice resulted in a significant hyperpolarizing shift in the resting membrane potential (RMP; Zobeiri et al., 2018), resembling the RMP of the TC neurons during deep SWS and increased possibility of bursting activity in these neurons (Zobeiri et al., 2018). Relevant is that the GABA containing TRN neurons in mice do not express TRIP8b ${ }^{-1-}$ (Heuermann et al., 2016; Zobeiri et al., 2018). Therefore, $\mathrm{I}_{\mathrm{h}}$-dependent changes in the intrinsic properties of TRN neurons can be ruled out. However, considering the significant increase in the directed CT coupling as found here in TRIP8 $\mathrm{b}^{-1-}$ mice, changes in the firing pattern of the TRN GABAergic neurons are expected. In fact, the large descending $\mathrm{SoSeCx}$ drive to the VPM in TRIP8b ${ }^{-1-}$ mice, next to a diminished CC drive, is the most prominent finding in this study.

This increased excitatory glutamatergic drive from cortex to thalamus would also stimulate GABAergic cells in the TRN. The cortical drive to the TRN is much stronger than the cortical drive to the VPM (Golshani et al., 2001), so that the inhibition of the TC neurons by the TRN can overcome the direct excitatory drive from CT cells. This mechanism of lateral inhibition of the TC neurons by TRN through direct GABAergic pathways has been described by Pinault (2004). Through increased lateral inhibition, the TC cells become hyperpolarized, irrespective of whether the mice are awake or asleep. We found earlier that in TRIP8b ${ }^{-1-}$ mice, the downregulation of $I_{h}$ in cortex and thalamus, as well as decreased cAMP signaling in the thalamus, is responsible for transformation of the normal physiological sleep- and wake oscillations to a new form of TCD characterized by a significant increase in slow $(\delta)$ frequency oscillations during episodes of AW. Within the TRN, most likely PV containing oscillatory cells involved in bursting, interconnected through electrical synapses, synchronize this inhibitory effect throughout the TRN, and inhibit and hyperpolarize multiple TC cells and cause the $\delta$-rage burst activity in the thalamus.

\section{Differences between methods}

Linear and nonlinear methods have been applied for causality analysis in neuroscience for many years (Gourévitch et al., 2006). Linear methods such as correlation and cross-spectrum analysis are frequently considered as robust, mostly due to long history of application and simplicity of calculation and explanation. These measures and some of their widespread nonlinear generalizations, like nonlinear association analysis (Pijn et al., 1989) and mutual information function (Kraskov et al., 2004), are nondirectional by design, but they are frequently used for detection of dominating direction of coupling by evaluating the time shifts between the two signals. However, recent studies showed that relying on time shifts for coupling direction can be unsafe and even misleading (Vakorin et al., 2013). Moreover, directed linear methods such as linear GC can indicate coupling direction spuriously due to signal similarities, due to insufficient sampling rate (Smirnov and Bezruchko, 2012), synchrony (Sysoev and Sysoeva, 2015), or signal nonstationarity (He and Maekawa, 2001).
Also, the usage of inappropriate nonlinear functions or mistakes in time-lag detection for vector-state reconstruction and for the construction of the prediction model (models are required for GC approach) can lead to wrong findings in coupling direction (Kornilov et al., 2016), even for nonlinear models. Therefore, to reduce the chance for spurious causalities and to increase the sensitivity of coupling analysis, the nonlinear adapted GC with specifically tuned models [we followed the recently developed method framework for model fitting to LFP data, as reported in Sysoeva and Medvedeva (2018) and Sysoeva et al. (2016a), but modified it to the specifics of the considered data] was used as a primary tool, and coherence analysis was used in addition.

As mentioned in the method section, both unconditional and conditional nlGC were used. Discrepancies between these two methods, reduced coupling estimates in case of conditional method, are suggestive of other than the direct coupling in some cases. In general, there were only minor differences in the outcomes between the two nlGC methods; an exception was CC connectivity in TRIP $8 \mathrm{~b}^{-1-}$ mice during SWS. This discrepancy might point toward other and perhaps more complicated communication between the $\mathrm{SoSeCx}$ and $\mathrm{MoCx}$ in these mice, particularly during SWS, or that

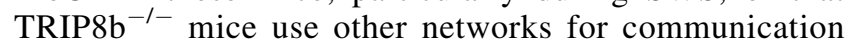
within the cortex when they shift from sleep to wake and vice versa. However, this issue regarding whether there are other nodes or hubs can only be studied and understood with more cortical and subcortical electrodes, or by combining white matter tractography with neurophysiology.

\section{Comparison of coherence and nIGC}

NlGC showed often a decrease in unconditional and conditional couplings between VPM and $\mathrm{SoSeCx}$ in both directions in sleep compared with AW in both WT and $\mathrm{TRIP}^{-1-}$ mice; coherence showed also the sleep induced decrease in WT, but for TRIP8b ${ }^{-1-}$ the difference was not significant. This could be due to a lower sensitivity of coherence compared with nlGC. NlGC also showed differences between WT and TRIP8b ${ }^{-1-}$ in the direction of the dominating TCT coupling during wakefulness: cortical influence to VPM is much larger in TRIP8b ${ }^{-1-}$ mice than in WT and also much stronger than in the reversed direction, once more suggesting that the bidirectional information flow between cortex and thalamus is seriously different from the WT.

Coherence cannot detect the direction of the couplings. A final difference between coherence and nlGC regards strain differences in the strength of couplings between cortex and thalamus. Coherence analyses showed that the genetically modified mice have a lower coherence during AW than the WT mice, while nlGC showed higher CT couplings. Since coherence is unable to detect the coupling direction, and since one cannot establish in which direction the impact is larger, the difference between the two methods does not come as a complete surprise. The difference can also be due to a large nonlinear contribution in the communication between thalamus and cortex and from some coupling specifics. For example, a coupling increase may lead to desynchronization and oscillation "death," as was demonstrated in a network for bidirectional inhibitory and asymmetric excitatory/inhibitory coupling (Bressloff and Coombes, 1998). If this is the case, coherence shows a significant decrease due to the absence of normal 
oscillations (only some subthreshold oscillations remain) in some neuronal subpopulation, while the causality becomes larger; this being the reason for the oscillation death.

Cortical coherence was clearly diminished during sleep for WT mice, but nlGC only showed insignificantly lower coupling from MoCx to SoSeCx. Different mechanisms of coupling, spurious detection of synchrony by coherence due to a finite time-series length, suboptimal parametrization (choosing not best values of model lag, prediction time and dimension) for nlGC method, since we were more focused on reducing false positives than on increasing sensitivity, or other factors could be reasons for these different results. This question has to be studied more with different tools.

\section{Conclusion}

Our network analyses showed that loss of a single protein involved in postsignaling processing might have large consequences for both $\mathrm{CC}$ and cortical-subcortical communication. Irrespective of the methods used, this study identified large and unambiguous differences in both $\mathrm{CC}$ and $\mathrm{CT}$ network interactions between TRIP8b ${ }^{-1-}$ and WT mice.

Our current and earlier findings (Zobeiri et al., 2018) indicated that altered properties of distinct ion channels determine alterations in rhythmicity and in CTC connectivity. It seems important to establish its clinical consequences, such as various modes of sensory processing and arousal reactions, next to the already described dysfunctions in nest-building behavior requiring finetuned sensory and motor functions, as well as the more gross motor functions as measured with the rotarod test (Lewis et al., 2011). More and other functional tests and phenotyping will give further information about the consequences of lacking the protein TRIP8b. Finally, disturbances in network interactions might also be present in other TCDs; our present work illustrates the usefulness of the various methods that were used here.

\section{Acknowledgments}

Ilya V. Sysoev is grateful to Russian Foundation for Basic Research (Grant No. 17-02-00307) for support of his work; Thomas Budde is grateful to Deutsches Forschungsgemeinschaft (DFG, BU 1019/15-1) and Interdisziplinäres Zentrum für Klinische Forschung Münster (IZKF, Bud3/001/16) for support. The authors thank Dr. M.V. Sysoeva for her advices regarding the use of conditional nonlinear Granger causality method and surrogate series construction.

\section{Author Disclosure Statement}

No competing financial interests exist.

\section{References}

Achermann P, Borbély AA. 1998. Temporal evolution of coherence and power in the human sleep electroencephalogram. J Sleep Res 7(Suppl. 1):36-41.

Baccalá LA, Sameshima K. 2001. Partial directed coherence: a new concept in neural structure determination. Biol Cybern 84:463-474.

Bressloff PC, Coombes S. 1998. desynchronization, mode locking, and bursting in strongly coupled integrate-and-fire oscillators. Phys Rev Lett 81:2168-2171.

Cain SM, John RT, Jones KL, Snutch TP. 2015. Thalamocortical neurons display suppressed burst-firing due to an enhanced Ih current in a genetic model of absence epilepsy. Pflügers Arch 467:1367-1382.

Chen Y, Rangarajan G, Feng J, Ding M. 2004. Analyzing multiple nonlinear time series with extended Granger causality. Phys Lett A 324:26-35.

Chung WK, Shinb M, Jaramillo TC, Leibel RL, LeDuc CA, Fischer SG, et al. 2009. Absence epilepsy in apathetic, a spontaneous mutant mouse lacking the $\mathrm{H}$ channel subunit, HCN2. Neurobiol Dis 33:499-508.

Coenen AM, Vendrik AJ. 1972. Determination of the transfer ratio of cat's geniculate neurons through quasi-intracellular recordings and the relation with the level of alertness. Exp Brain Res 14:227-242.

Datunashvili M, Chaudhary R, Zobeiri M, Lüttjohann A, Mergia E, Baumann A, et al. 2018. Modulation of hyperpolarizationactivated inward current and thalamic activity modes by different cyclic nucleotides. Front Cell Neurosci 12:369.

De Ridder D, Vanneste S, Langguth B, Llinas RR. 2015. Thalamocortical dysrhythmia: a theoretical update in tinnitus. Front Neurol 6:124.

Fogerson PM, Huguenard JR. 2016. Tapping the brakes: cellular and synaptic mechanisms that regulate thalamic oscillations. Neuron 92:687-704.

Golshani P, Liu X-B, Jones EG. 2001. Differences in quantal amplitude reflect GluR4-subunit number at corticothalamic synapses on two populations of thalamic neurons. Proc Natl Acad Sci U S A 98:4172-4177.

Gourévitch B, Le Bouquin-Jeannès R, Faucon G. Linear and nonlinear causality between signals: methods, examples and neurophysiological applications. Biol Cybern 95:349-369.

Guevara MA, Lorenzo I, Arce C, Ramos J, Corsi-Cabrera M. 1995. Inter- and intrahemispheric EEG correlation during sleep and wakefulness. Sleep 18:257-265.

He C, Chen F, Li B, Hu Z. 2014. Neurophysiology of HCN channels: from cellular functions to multiple regulations. Prog Neurobiol 112:1-23.

He Z, Koichi M. 2001. On spurious Granger causality. Econ Lett 73:307-313.

Heuermann RJ, Jaramillo TC, Ying S-W, Suter BA, Lyman KA, Han Y, et al. 2016. Reduction of thalamic and cortical Ih by deletion of TRIP8b produces a mouse model of human absence epilepsy. Neurobiol Dis 85:81-92.

Huber R, Deboer T, Tobler I. 2000. Topography of EEG dynamics after sleep deprivation in mice. $\mathbf{J}$ Neurophysiol 84:1888-1893.

Kole, MHP, Bräuer AU, Stuart GJ. 2007. Inherited cortical HCN1 channel loss amplifies dendritic calcium electrogenesis and burst firing in a rat absence epilepsy model. J Physiol 578:507-525.

Kornilov MV, Sysoev IV. 2013. Influence of the choice of the model structure for working capacity of nonlinear Granger causality approach. Izv VUZ Appl Nonlinear Dyn 21:74-87.

Kornilov MV, Medvedeva TM, Bezruchko BP, Sysoev IV. 2016. Choosing the optimal model parameters for Granger causality in application to time series with main timescale. Chaos Solitons Fractals 82:11-21.

Kraskov A, Stögbauer H, Grassberger P. 2004. Estimating mutual information. Phys Rev E 69:066138.

Kugiumtzis D. 1996. State space reconstruction parameters in the analysis of chaotic time series - the role of the time window length. Physica D 95:13-28.

Lewis AS, Vaidya SP, Blaiss CA, Liu Z, Stoub TR, Brager DH, et al. 2011. Deletion of the hyperpolarization-activated cyclic nucleotide-gated channel auxiliary subunit TRIP8b impairs 
hippocampal Ih localization and function and promotes antidepressant behavior in mice. J Neurosci 31:7424-7440.

Llinás RR, Ribary U, Jeanmonod D, Kronberg E, Mitra PP. 1999. Thalamocortical dysrhythmia: a neurological and neuropsychiatric syndrome characterized by magnetoencephalography. Proc Natl Acad Sci U S A 96:15222-15227.

Llinás RR, Steriade M. 2006. Bursting of thalamic neurons and states of vigilance. J Neurophysiol 95:3297-3308.

Llinás RR, Urbano FJ, Leznik E, Ramírez RR, van Marle HJF. 2005. Rhythmic and dysrhythmic thalamocortical dynamics: GABA systems and the edge effect. Trends Neurosci 28:325-333.

Ludwig A, Budde T, Stieber J, Moosmang S, Wahl C, Holthoff K, et al. 2003. Absence epilepsy and sinus dysrhythmia in mice lacking the pacemaker channel HCN2. EMBO J 22:216-224.

Maris E, Oostenveld R. 2007. Nonparametric statistical testing of EEG- and MEG-data. J Neurosci Methods 164:177-190.

Medvedeva TM, Lüttjohann AK, van Luijtelaar G, Sysoev IV. 2016. Evaluation of non-linear properties of epileptic activity using largest Lyapunov exponent. Proc SPIE 9917:991724.

Pal D, Silverstein BH, Lee H, Mashour GA. 2016. Neural correlates of wakefulness, sleep, and general anesthesia. Anesthesiology 125:929-942.

Picchioni D, Pixa ML, Fukunaga M, Carr WS, Horovitz SG, Braun AR, Duyn JH. 2014. Decreased connectivity between the thalamus and the neocortex during human nonrapid eye movement sleep. Sleep 37:387-397.

Pijn J, Vijn P, Lopes da Silva F, van Emde Boas W, Blanes W. 1989. The use of signal-analysis for the location of an epileptogenic focus: a new approach. Adv Epileptol 17:272-276.

Pinault D. 2004. The thalamic reticular nucleus: structure, function and concept. Brain Res Rev 46:1-31.

Proske JH, Jeanmonod D, Verschure PFMJ. 2011. A computational model of thalamocortical dysrhythmia. Eur J Neurosci 33:1281-1290.

Schelter B, Timmer J, Eichler M. 2009. Assessing the strength of directed influences among neural signals using renormalized partial directed coherence. J Neurosci Methods 179:121-130.

Schwarz G. 1978. Estimating the dimension of a model. Ann Statist 6:461-464.

Sherman SM. 2017. Functioning of circuits connecting thalamus and cortex. Compr Physiol 7:713-739.

Smirnov DA, Bezruchko BP. 2012. Spurious causalities due to low temporal resolution: towards detection of bidirectional coupling from time series. Europhys Lett 100:10005.

Smirnov DA, Sidak EV, Bezruchko BP. 2013. Detection of coupling between oscillators with analytic tests for significance. Eur Phys J Spec Top 222:2441-2451.

Spoormaker VI, Schröter MS, Gleiser PM, Andrade KC, Dresler M, Wehrle R, et al. 2010. Development of a large-scale functional brain network during human non-rapid eye movement sleep. J Neurosci 30:11379-11387.

Steriade M, Datta S, Pare D, Oakson G, Curro Dossi RC. 1990. Neuronal activities in brain-stem cholinergic nuclei related to tonic activation processes in thalamocortical systems. J Neurosci 10:2541-2559.

Steriade M, McCormick DA, Sejnowski TJ. 1993. Thalamocortical oscillations in the sleeping and aroused brain. Science 262:679-685.

Sysoev IV, Sysoeva MV. 2015. Detecting changes in coupling with Granger causality method from time series with fast transient processes. Physica D 309:9-19.

Sysoeva MV, Kuznetsova GD, Sysoev IV. 2016a. The modeling of rat EEG signals in absence epilepsy in the analysis of brain connectivity. Biophysics 61:661-669.
Sysoeva MV, Lüttjohann AK, van Luijtelaar G, Sysoev IV. 2016b. Dynamics of directional coupling underlying spikewave discharges. Neuroscience 314:75-89.

Sysoeva MV, Medvedeva TM. 2018. Optimization of Granger causation method parameters for the study of limbic epilepsy. Izvestiya VUZ Appl Nonlinear Dynam 26:39-62.

Sysoeva MV, Sysoev IV. 2012. Mathematical modeling of encephalogram dynamics during epileptic seizure. Techn Phys Lett 38:151-154.

Theiler J, Eubank S, Longtin A, Galdrikian B, Doyne Farmer J. 1992. Testing for nonlinearity in time series: the method of surrogate data. Physica D 58:77-94.

Timofeev I, Bazhenov M. 2005. Mechanisms and biological role of thalamocortical oscillations. In: Columbus F (ed.) Trends in Chronobiology Research. New York: Nova Science Publishers, Inc.; p. 1.

Timofeev I, Contreras D, Steriade M. 1996. Synaptic responsiveness of cortical and thalamic neurones during various phases of slow sleep oscillation in cat. J Phys 494:265-278.

Vakorin VA, Krakovska MB, Bezgin OG, McIntosh R. 2013. Confounding effects of phase delays on causality estimation. PLoS One 8:e53588.

van Der Walt S, Colbert SC, Varoquaux G. 2011. The NumPy array: a structure for efficient numerical computation. Comput Sci Eng 2:22-30.

van Luijtelaar G, Wilde M, Citraro R, Scicchitano F, van Rijn CM. 2012. Does antiepileptogenesis affect sleep in genetic epileptic rats? Int J Psychophysiol 85:49-54.

White BR, Bauer AQ, Snyder AZ, Schlaggar BL, Lee JM, Culver JP. 2011. Imaging of functional connectivity in the mouse brain. PLoS One 6:e16322.

Woodward ND, Cascio CJ. 2015. Resting-state functional connectivity in psychiatric disorders. JAMA Psychiatry 72:743-744.

Woodward ND, Karbasforoushan H, Heckers S. 2012. Thalamocortical dysconnectivity in schizophrenia. Am J Psychiatry 169:1092-1099.

Zhang D, Guo L, Hu X, Li K, Zhao Q, Liu T. 2012. Increased cortico-subcortical functional connectivity in schizophrenia. Brain Imaging Behav 6:27-35.

Zhang Y, Llinas RR, Lisman JE. 2009. Inhibition of NMDARs in the nucleus reticularis of the thalamus produces delta frequency bursting. Front Neural Circuits 3:20.

Zobeiri M, Chaudhary R, Datunashvili M, Heuermann RJ, Lüttjohann A, Narayanan V, et al. 2018. Modulation of thalamocortical oscillations by TRIP8b, an auxiliary subunit for HCN channels. Brain Struct Funct 223:1537-1564.

Address correspondence to: Ilya $V$. Sysoev Saratov Branch of Kotel'nikov Institute of Radio Engineering and Electronics of RAS Saratov 410019 Russia

E-mail: ivssci@gmail.com

Mehrnoush Zobeiri Institute of Physiology I (Neurophysiology) Wesfälische Wilhelms University Münster 48149

Germany

E-mail: zobeiri@uni-muenster.de $($ Appendix follows $\rightarrow$ ) 


\section{Appendix}

To make sure that the results of bivariate or unconditional nonlinear Granger causality (nlGC) analyses were not affected by mediated multivariate coupling, the conditional nlGC method was applied following Chen et al. (2004), but with nonuniform embedding as proposed earlier (Sysoeva and Sysoev, 2012), and adapted to the frequency properties of the LFP signals. The models that were used for the conditional nlGC had the same dimension and polynomial order as were used for unconditional causality.

We also generated the same number of surrogate series as for unconditional nlGC by random shuffling of different episodes (three different episodes here in comparison with two for unconditional nlGC). It is expected that there will be no significant increase in coupling reported by conditional nlGC in comparison with unconditional nlGC. Such an increase would be hard to explain, considering that the inclusion of a third time series might also explain some couplings, and as a consequence, a diminishment in the original coupling might be expected. The results of conditional nlGC show the following differences in the results of unconditional nlGC (compare Figs. 3 and 4).

First, no significant increase was found in any of the pairs, and most pairs kept their couplings or showed less coupling. Some of the discrepancies between the methods can be ascribed to the added error variance of the third time series, the model's imperfection, the finite time-series length, and the sampling rate. Second, a significant reduction was found in wild-type (WT) mice. Here, the ventral posterior medial nucleus of the thalamus (VPM) $\rightarrow$ somatosensory cortex (SoSeCx) couplings were no longer leading, in addition to that the motor cortex (MoCx) $\rightarrow$ SoSeCx coupling during wakefulness lost its leading position. Third, the most pronounced difference between the two methods was found in the cortical pairs in both directions during sleep in TRIP8b ${ }^{-1-}$ mice: the SoSeCx $\rightarrow$ MoCx was no longer leading, and a significantly lower cortical coupling was found in TRIP8 $\mathrm{b}^{-{ }_{-}}$mice during wakefulness as well as during slow-wave sleep (SWS) compared with WT mice. Nevertheless, the reduced coupling in the multivariate analyses compared with the bivariate analysis strongly suggests that these cortical couplings are to a large extent indirect. The latter, to be partly mediated (about one half) by indirect coupled pathways, also seems true for the $\mathrm{VPM} \rightarrow$ SoSeCx coupling in WT mice during active wakefulness. The strongest directed coupling, found between SoSeCx and VPM both during wakefulness and during deep SWS in TRIP8b ${ }^{-1-}$ mice, was unaffected by the method. It still showed a leading role for the SoSeCx in coupling to the VPM and a reduction in coupling strength during SWS in both directions. 